PROCEEDINGS OF THE

AMERICAN MATHEMATICAL SOCIETY

Volume 138, Number 11, November 2010, Pages 4109-4117

S 0002-9939(2010)10512-9

Article electronically published on June 11, 2010

\title{
CORRESPONDENCES OF HYPERSURFACES IN HYPERBOLIC POINCARÉ MANIFOLDS AND CONFORMALLY INVARIANT PDES
}

\author{
VINCENT BONINI, JOSÉ M. ESPINAR, AND JIE QING
}

(Communicated by Matthew J. Gursky)

\begin{abstract}
On a hyperbolic Poincaré manifold, we derive an explicit relationship between the eigenvalues of Weyl-Schouten tensor of a conformal representative of the conformal infinity and the principal curvatures of the level sets of the associated geodesic defining function. This considerably simplifies the arguments and generalizes the results of Gálvez, Mira and the second author. In particular, we obtain the equivalence between Christoffel-type problems for hypersurfaces in a hyperbolic Poincaré manifold and scalar curvature problems on the conformal infinity.
\end{abstract}

\section{INTRODUCTION}

The relationship between the geometry of a conformally compact space and the geometry of its conformal infinity has been of recent interest in both physical and mathematical communities. The current interest in such association is motivated primarily by the AdS/CFT correspondence. In fact, the study of such connections date back to the 1980's in the seminal paper of Fefferman and Graham [7].

Recently a correspondence between the geometry of hyperbolic space $\mathbb{H}^{n+1}$ and the conformal geometry of the round sphere $\mathbb{S}^{n}$ was exhibited in [5] in the context of the hypersurface geometry of $\mathbb{H}^{n+1}$ and curvature prescription problems on $\mathbb{S}^{n}$ in the conformal class of the round metric. Of particular interest is the Christoffel problem for hypersurfaces in hyperbolic space $\mathbb{H}^{n+1}$ where one is asked to find a hypersurface $\Sigma^{n} \subset \mathbb{H}^{n+1}$ with prescribed mean of the curvature radii. In [5] the relevant notions of the hyperbolic Gauss map and the hyperbolic principal curvature radii are developed using the ambient structure of the hyperboloid model of $\mathbb{H}^{n+1}$. Moreover, [5] exhibits a precise relation between Christoffel-type problems for immersed hypersurfaces in $\mathbb{H}^{n+1}$ and scalar curvature prescription problems of conformal geometry on $\mathbb{S}^{n}$.

In this paper we take a viewpoint more reflective of conformal geometry, which allow us to considerably simplify the arguments and generalize the correspondences in [5]. A hyperbolic Poincaré manifold is a conformally compact hyperbolic manifold. From the work of $[\underline{8}$, given a representative $\gamma \in[\hat{g}]$ of the conformal infinity

Received by the editors October 16, 2009 and, in revised form, February 12, 2010.

2010 Mathematics Subject Classification. Primary 53C30, 53C40; Secondary 58J05.

Key words and phrases. Hyperbolic Poincaré manifold, hypersurfaces, second fundamental form, Schouten tensor, conformally invariant PDE.

(C)2010 American Mathematical Society Reverts to public domain 28 years from publication 
of a hyperbolic Poincaré manifold $\left(X^{n+1}, g\right)$ and its associated geodesic defining function $r$, we may write the metric in the normal form

$$
g=r^{-2}\left(d r^{2}+g_{r}\right)
$$

with

$$
g_{r}=\gamma-r^{2} P_{\gamma}+\frac{r^{4}}{4} Q\left(P_{\gamma}\right),
$$

where $Q\left(P_{\gamma}\right)_{i j}=\gamma^{k l}\left(P_{\gamma}\right)_{i k}\left(P_{\gamma}\right)_{j l}$ and where $P_{\gamma}$ is the Weyl-Schouten tensor of $\gamma$ when $n \geq 3$ and is a symmetric 2 -tensor on $M$ satisfying

$$
\gamma^{i j}\left(P_{\gamma}\right)_{i j}=\frac{R_{\gamma}}{2} \quad \text { and } \quad \gamma^{j k}\left(P_{\gamma}\right)_{i j, k}=\left(R_{\gamma}\right)_{, i}
$$

when $n=2$. A straightforward calculation based on (2) yields a generalization of the relation in [5] between the eigenvalues of the Schouten tensor of the horospherical metric and the hyperbolic principal curvature radii of the level sets of the associated geodesic defining function. We recall that the second fundamental form of a hypersurface $\Sigma$ in $X^{n+1}$ with respect to an orientation induced by a choice a normal direction $N$ is defined to be

$$
I I=-\frac{1}{2} \mathcal{L}_{N} g,
$$

where $\mathcal{L}$ is the Lie derivative. In our convention, for instance, the principal curvature of a unit sphere in Euclidean space with the orientation induced by the inward normal direction is 1 .

Theorem 1.1. Suppose that $\left(X^{n+1}, g\right)$ is a hyperbolic Poincaré manifold and let $\gamma$ be a representative of its conformal infinity $\left(M^{n},[\hat{g}]\right)$ with associated geodesic defining function $r$. Then the eigenvalues $\lambda_{i}$ of the tensor $P_{\gamma}$ in the expansion (2) satisfy

$$
1-\frac{r^{2}}{2} \lambda_{i}=\frac{2}{1-\kappa_{i}},
$$

where $\kappa_{i}=\kappa_{i}(r)$ denotes the $i^{\text {th }}$ outward principal curvature on the level sets of the geodesic defining function $r$ and $\frac{2}{1-\kappa_{i}}$ is considered to be the $i^{\text {th }}$ hyperbolic principal curvature radius.

As studied in [5], when $n \geq 3$, the relationship (41) in Theorem [1.1] can be used to turn questions regarding foliations near the conformal infinity by particular classes of hypersurfaces in hyperbolic Poincaré manifolds into questions regarding the conformal geometry of the conformal infinity and vice versa. For example, taking the trace of (4), one finds that

$$
R_{\gamma}=\frac{4(n-1)}{r^{2}}\left(n-\sum_{i=1}^{n} \frac{2}{1-\kappa_{i}}\right) .
$$

Therefore, finding a foliation by hypersurfaces with constant mean of the hyperbolic curvature radii is equivalent to finding a constant scalar curvature metric on the conformal infinity. Hence, due to the resolution of the Yamabe problem we have the following corollary. 
Corollary 1.1. Suppose that $\left(X^{n+1}, g\right)$ is a hyperbolic Poincaré manifold. Then there always exists a foliation of hypersurfaces of constant mean of the hyperbolic curvature radii near the infinity. Such foliations are parameterized by geodesic defining functions $r$ associated with constant scalar curvature $S$ representatives of the conformal infinity, and the mean of the hyperbolic curvature radii of the foliation is given by

$$
\frac{1}{n} \sum_{i=1}^{n} \frac{2}{1-\kappa_{i}}=1-\frac{r^{2}}{4 n(n-1)} S
$$

Moreover, if the conformal infinity $(M,[\hat{g}])$ of $\left(X^{n+1}, g\right)$ has negative Yamabe invariant, then such foliations are unique.

More generally, the relationship (44) can similarly be applied to the generalized Yamabe or $\sigma_{k}$ curvature problem to give foliations of certain hyperbolic Poincaré manifolds by hypersurfaces with constant linear combinations or rational functions of generalized mean curvatures. For $1 \leq k \leq n$ and $\lambda=\left(\lambda_{1}, \ldots, \lambda_{n}\right) \in \mathbb{R}^{n}$, let

$$
\sigma_{k}(\lambda):=\sum_{i_{1}<\cdots<i_{k}} \lambda_{i_{1}} \cdots \lambda_{i_{k}}
$$

denote the $k^{\text {th }}$ elementary symmetric function on $\mathbb{R}^{n}$. Let $\Gamma_{k}$ denote the connected component of

$$
\left\{\lambda \in \mathbb{R}^{n} \mid \sigma_{k}(\lambda)>0\right\}
$$

containing the positive cone $\left\{\lambda \in \mathbb{R}^{n} \mid \lambda_{1}, \ldots, \lambda_{n}>0\right\}$. Given a representative $g_{0}$ of the conformal infinity $\left(M^{n},[\hat{g}]\right)$ of a hyperbolic Poincaré manifold $\left(X^{n+1}, g\right)$, we denote the eigenvalues $\lambda=\left(\lambda_{1}, \ldots, \lambda_{n}\right)$ of the the Schouten tensor $P_{g_{0}}$ by $\lambda\left(P_{g_{0}}\right)$ and the $k^{t h}$ elementary symmetric function of the eigenvalues of the Schouten tensor $P_{g_{0}}$ by $\sigma_{k}\left(P_{g_{0}}\right)$. Moreover, if $\tilde{g}_{0}=e^{2 \phi_{0}} g_{0}$ is a conformally related metric on $M$, then we denote the $k^{\text {th }}$ elementary symmetric function of the eigenvalues of the Schouten tensor $P_{\tilde{g}_{0}}$ corresponding to $\tilde{g}_{0}$ by $\sigma_{k}\left(P_{\tilde{g}_{0}}\right)$. Applying the works of [10] [11 [13, it follows from that fact that $M$ is compact and locally conformally flat, that for $n \geq 3$, if $\lambda\left(P_{g_{0}}\right) \in \Gamma_{k}$, then there exists a smooth positive function $\phi_{0}$ on $M$ such that $\tilde{g}_{0}=e^{2 \phi_{0}} g_{0}$ with

$$
\lambda\left(P_{\tilde{g}_{0}}\right) \in \Gamma_{k} \quad \text { and } \quad \sigma_{k}\left(P_{\tilde{g}_{0}}\right)=1 .
$$

In light of (4), (7) and the observations above, we have the following corollary.

Corollary 1.2. For $n \geq 3$, let $\left(X^{n+1}, g\right)$ be a hyperbolic Poincaré manifold with conformal infinity $\left(M^{n},[\hat{g}]\right)$. Suppose that there exists a metric $g_{0} \in[\hat{g}]$ with $\lambda\left(P_{g_{0}}\right) \in \Gamma_{k}$ for some $1 \leq k \leq n$. Then there exists a foliation near $M$ parameterized by a geodesic defining function $r$ associated to a conformal metric $\tilde{g}_{0} \in\left[g_{0}\right]$ with constant $\sigma_{k}$ curvature $\sigma_{k}\left(P_{\tilde{g}_{0}}\right)=1$ such that the level sets of $r$ have outward principal curvatures $\kappa_{i}=\kappa_{i}(r)$ satisfying

$$
\sum_{i_{1}<\cdots<i_{k}} \frac{1+\kappa_{i_{1}}}{1-\kappa_{i_{1}}} \cdot \frac{1+\kappa_{i_{2}}}{1-\kappa_{i_{2}}} \cdots \frac{1+\kappa_{i_{k}}}{1-\kappa_{i_{k}}}=\left(\frac{r^{2}}{2}\right)^{k} .
$$

To reconcile the two different approaches in [5] and in [7] and [8], we will show that the horospherical metric associated to a horospherical ovaloid in hyperbolic space $\mathbb{H}^{n+1}$ can be realized as a representative of the conformal infinity $\left(\mathbb{S}^{n},\left[g_{0}\right]\right)$ of hyperbolic space $\left(\mathbb{H}^{n+1}, g_{\mathbb{H}}\right)$. This is because a horospherical ovaloid in hyperbolic 
space $\mathbb{H}^{n+1}$ determines a geodesic defining function $r$ for the infinity $\mathbb{S}^{n}$ of $\mathbb{H}^{n+1}$, where $r=e^{-s}$ and $s$ is the hyperbolic distance to the horospherical ovaloid. A similar idea was used in the early works of Epstein [4].

This paper is organized as follows. In Section 2 we introduce hyperbolic Poincaré manifolds and the asymptotic expansion (2) from [8]. Then we will do the straightforward calculation to prove Theorem [1.1. In Section 3 we introduce the notion of the horospherical metric associated to a horospherical ovaloid in $\mathbb{H}^{n+1}$ and we relate such horospherical metrics to representatives of the conformal infinity. This observation allows us to put the two constructions in [5] and in [7] and [8] in the same light.

\section{The CAlculation}

In this section we carry out the straightforward calculation to prove our main theorem. Let $X^{n+1}$ denote the interior of a smooth compact manifold $\bar{X}^{n+1}$ with boundary $\partial X=M^{n}$. A smooth function $r: \bar{X} \rightarrow \mathbb{R}$ is said to be a defining function for $M$ in $X$ if $r>0$ in $X, r=0$ on $M$ and $d r \neq 0$ on $M$. A Riemannian metric $g$ on $X$ is then said to be conformally compact if for a defining function $r$ for $M$, the conformal metric $\bar{g}=r^{2} g$ extends to a metric on $\bar{X}$. The metric $\bar{g}$ restricted to $T M$ induces a metric $\hat{g}$ on $M$, which rescales by conformal factor upon change in defining function and therefore defines a conformal structure $(M,[\hat{g}])$ on $M$ called the conformal infinity of $(X, g)$. A complete Riemannian manifold $\left(X^{n+1}, g\right)$ is said to be asymptotically hyperbolic if $g$ is conformally compact and $|d r|_{\bar{g}}^{2}=1$ on $M$ for a defining function $r$ for $M$ in $X$. We recall from $[9]$ and [12] that there is a unique geodesic defining function $r$ associated with a representative in the conformal infinity of an asymptotically hyperbolic manifold. Moreover, such choice of coordinates yields the normal form

$$
g=r^{-2}\left(d r^{2}+g_{r}\right)
$$

with formal asymptotic expansion

$$
g_{r}=g_{0}+r g_{1}+r^{2} g_{2}+\cdots
$$

where the coefficients $g_{j}$ are symmetric 2-tensors on $M$. The asymptotic expansions described above are fundamental in many works concerning the geometry and topology of conformally compact manifolds in the spirit of the AdS/CFT correspondence (see for example [1], 2], 3], 6], [14]).

In this paper we focus on a class of manifolds that serve as the prototypical models of asymptotically hyperbolic manifolds known as hyperbolic Poincaré manifolds, which are conformally compact hyperbolic manifolds. Suppose that $\left(X^{n+1}, g\right)$ is a hyperbolic Poincaré manifold and $\left(M^{n},[\hat{g}]\right)$ is its conformal infinity. Let $\gamma$ be a representative of the conformal infinity and let $r$ be the geodesic defining function associated to $\gamma$ so that $g$ has the normal form

$$
g=r^{-2}\left(d r^{2}+g_{r}\right)
$$

near $M$. Due to the fact that hyperbolic Poincaré manifolds are exactly conformally compact Einstein manifolds with locally conformally flat conformal infinities, we recall from [8] that

$$
g_{r}=\gamma-r^{2} P_{\gamma}+\frac{r^{4}}{4} Q\left(P_{\gamma}\right)
$$


where

$$
Q\left(P_{\gamma}\right)_{i j}=\gamma^{k l}\left(P_{\gamma}\right)_{i k}\left(P_{\gamma}\right)_{j l}
$$

and for $n \geq 3$,

$$
P_{\gamma}=\frac{1}{n-2}\left(R i c_{\gamma}-\frac{R_{\gamma}}{2(n-1)} \gamma\right)
$$

is the Schouten tensor of $\gamma$. For $n=2, P_{\gamma}$ is a symmetric 2-tensor on $M$ satisfying

$$
\gamma^{i j}\left(P_{\gamma}\right)_{i j}=\frac{R_{\gamma}}{2} \quad \text { and } \quad \gamma^{j k}\left(P_{\gamma}\right)_{i j, k}=\left(R_{\gamma}\right)_{, i}
$$

Then the level sets of $r$ give a foliation near $M$ with induced metric

$$
I_{r}=r^{-2} g_{r}=r^{-2} \gamma-P_{\gamma}+\frac{r^{2}}{4} Q\left(P_{\gamma}\right)
$$

and outward pointing normal $N_{r}=-r \partial_{r}$, where $\partial_{r}:=\nabla_{\bar{g}} r$. Hence, the level sets of $r$ have second fundamental form, according to our definition (3),

$$
I I_{r}=\frac{1}{2} r \partial_{r}\left(r^{-2} g_{r}\right)=-r^{-2} \gamma+\frac{r^{2}}{4} Q\left(P_{\gamma}\right)
$$

Now let $\left\{e_{1}, \ldots, e_{n}\right\}$ denote an orthonormal basis with respect to $\gamma$ that diagonalizes the tensor $P_{\gamma}$. Then

$$
\gamma\left(e_{i}, e_{j}\right)=\delta_{i j} \quad \text { and } \quad P_{\gamma}\left(e_{i}, e_{j}\right)=\lambda_{i} \delta_{i j},
$$

where $\lambda_{i}$ denotes the $i^{\text {th }}$ eigenvalue of the tensor $P_{\gamma}$. Moreover,

$$
I_{r}\left(e_{i}, e_{j}\right)=r^{-2}\left(1-\frac{r^{2}}{2} \lambda_{i}\right)^{2} \delta_{i j}
$$

and

$$
I I_{r}\left(e_{i}, e_{j}\right)=-r^{-2}\left(1-\frac{r^{2}}{2} \lambda_{i}\right)\left(1+\frac{r^{2}}{2} \lambda_{i}\right) \delta_{i j}
$$

Therefore,

$$
\left(I_{r}^{-1} I I_{r}\right)\left(e_{i}, e_{j}\right)=-\frac{1+\frac{r^{2}}{2} \lambda_{i}}{1-\frac{r^{2}}{2} \lambda_{i}} \delta_{i j}
$$

But the Weingarten matrix $I_{r}^{-1} I I_{r}$ on the level sets of $r$ satisfies

$$
\left(I_{r}^{-1} I I_{r}\right)\left(e_{i}, e_{j}\right)=\kappa_{i}^{r} \delta_{i j}
$$

where $\kappa_{i}^{r}$ denotes the $i^{\text {th }}$ principal curvature of a level set of $r$ with respect to the outward direction. Hence,

$$
\kappa_{i}^{r}=-\frac{2}{1-\frac{r^{2}}{2} \lambda_{i}}+1
$$

so that

$$
1-\frac{r^{2}}{2} \lambda_{i}=\frac{2}{1-\kappa_{i}^{r}}
$$

which establishes Theorem 1.1 . 


\section{HOROSPHERICAL METRICS}

In this section we introduce the horospherical metric on the space of all horospheres as a parametrization of a neighborhood of the infinity of hyperbolic space. Our goal is to relate horospherical metrics to representatives of the conformal infinity and to reconcile the two constructions in [5] and in [7] and [8].

Consider the hyperboloid model of hyperbolic space

$$
\mathbb{H}^{n+1}=\left\{\left.(x, t) \in \mathbb{R}^{n+1,1}|| x\right|^{2}-t^{2}=-1, t>0\right\},
$$

where $\mathbb{R}^{n+1,1}$ denotes Minkowski spacetime equipped with the Lorentz metric $g_{\mathbb{L}}=$ $-|d t|^{2}+|d x|^{2}$. Horospheres in $\mathbb{H}^{n+1}$ are intersections of degenerate affine hyperplanes of $\mathbb{R}^{n+1,1}$ with $\mathbb{H}^{n+1}$ and can be uniquely characterized by their points at infinity $x \in \mathbb{S}^{n}$, which are the null directions inside the hyperplanes, and the signed hyperbolic distance $\alpha$ from the horosphere to the vertex $e_{n+2} \in \mathbb{H}^{n+1}$, where $\alpha$ is positive if $e_{n+2}$ is inside a given horosphere and negative otherwise. Accordingly, one can identify the space of horospheres in $\mathbb{H}^{n+1}$ with $\mathbb{S}^{n} \times \mathbb{R}$ and endow the space of horospheres with a natural degenerate metric $\langle\cdot, \cdot\rangle_{\infty}=e^{2 \alpha} g_{0}$, where $g_{0}$ is the standard round metric on $\mathbb{S}^{n}$.

Now suppose

$$
\phi: \Sigma^{n} \rightarrow \mathbb{H}^{n+1}
$$

is an immersed oriented hypersurface and let

$$
\eta: \Sigma^{n} \rightarrow \mathbb{S}_{1}^{n+1}
$$

denote the Lorentzian unit normal map taking values in de-Sitter spacetime

$$
\mathbb{S}_{1}^{n+1}=\left\{\left.(x, t) \in \mathbb{R}^{n+1,1}|| x\right|^{2}-t^{2}=1\right\} .
$$

From the map

$$
\psi:=\phi+\eta: \Sigma^{n} \rightarrow \mathbb{N}_{+}^{n+1},
$$

taking values in the positive light-cone

$$
\mathbb{N}_{+}^{n+1}=\left\{\left.(x, t) \in \mathbb{R}^{n+1,1}|| x\right|^{2}-t^{2}=0, t>0\right\}
$$

one defines the hyperbolic Gauss map as the direction of the light-cone map (9) in $\mathbb{S}^{n}$. One finds that the light-cone map of horospheres is constant for the inward orientation and that parallel horospheres correspond to collinear vectors in $\mathbb{N}_{+}^{n+1}$. Hence, one can also identify the space of horospheres in $\mathbb{H}^{n+1}$ with $\mathbb{N}_{+}^{n+1}$. Moreover, it is easily seen that the horospherical metric on the space of all horospheres is exactly the same as the induced metric on the light-cone from the Lorentz metric $g_{\mathbb{L}}$ of Minkowski spacetime.

One can therefore realize the horospherical metric associated to a horospherical ovaloid in $\mathbb{H}^{n+1}$, that is, a compact hypersurface $\Sigma^{n} \subseteq \mathbb{H}^{n+1}$ for which the Gauss map is regular, as the pull-back by the light-cone map $\psi$ of the induced metric on the hypersurface as viewed in the positive light-cone $\mathbb{N}_{+}^{n+1}$. We recall from [5] that a compact immersed hypersurface is said to be a horospherical ovaloid in $\mathbb{H}^{n+1}$ if it can be oriented so that it is horospherically convex at every point and that an oriented hypersurface in $\mathbb{H}^{n+1}$ is horospherically convex at a point if and only if all the principal curvatures at the point are simultaneously less than 1 or greater than 1.

Alternatively, one can define the horospherical metric as in [15] by

$$
h:=I_{\Sigma}-2 I I_{\Sigma}+I I I_{\Sigma},
$$


where $I_{\Sigma}, I I_{\Sigma}$ and $I I I_{\Sigma}$ are respectively the first, second and third fundamental forms of $\Sigma$ in $\mathbb{H}^{n+1}$. In 5] Espinar, Gálvez and Mira view the image of the light cone map (9) as a co-dimension 2 hypersurface in Minkowoski spacetime and derive a relation between the principal curvatures of an immersed hypersurface in $\mathbb{H}^{n+1}$ and the eigenvalues of the Schouten tensor of its associated horospherical metric. In order to connect the work of $[5]$ with ours in the context of conformal geometry, we compute the horospherical metric as defined in (10). Given a hyperbolic Poincaré manifold $\left(X^{n+1}, g\right)$ and a representative $\gamma$ of its conformal infinity $\left(M^{n},[\hat{g}]\right)$, we first compute the third fundamental form on level sets determined by the associated geodesic defining function $r$. A straightforward computation gives

$$
\begin{aligned}
& I I I_{r}\left(\partial_{i}, \partial_{j}\right)=I_{r}\left(\nabla_{\partial_{i}} N_{r}, \nabla_{\partial_{j}} N_{r}\right)=I_{r}\left(\nabla_{\partial_{i}} r \partial_{r}, \nabla_{\partial_{j}} r \partial_{r}\right) \\
& =r^{-2} g_{i j}^{r}-r^{-1} \partial_{r} g_{i j}^{r}+\frac{1}{4} g_{r}^{p q} \partial_{r} g_{i p}^{r} \partial_{r} g_{j q}^{r} .
\end{aligned}
$$

In terms of an orthonormal basis $\left\{e_{1}, \ldots, e_{n}\right\}$ with respect to $\gamma$ that diagonalizes the tensor $P_{\gamma}$, it follows that

$$
I I I_{r}\left(e_{i}, e_{j}\right)=r^{-2}\left(1+\frac{r^{2}}{2} \lambda_{i}\right)^{2} \delta_{i j} .
$$

Therefore, the horospherical metric associated to a level set of a geodesic defining function $r$ is

$$
h\left(e_{i}, e_{j}\right)=I_{r}\left(e_{i}, e_{j}\right)-2 I I_{r}\left(e_{i}, e_{j}\right)+I I I_{r}\left(e_{i}, e_{j}\right)=4 r^{-2} \delta_{i j} .
$$

Thus, given a conformal representative $\gamma$ of the conformal infinity $\left(M^{n},[\hat{g}]\right)$ of a hyperbolic Poincaré manifold $\left(X^{n+1}, g\right)$ and its associated geodesic defining function $r$, the horospherical metrics associated to the level sets of $r$ are given by $h=4 r^{-2} \gamma$. On the other hand, given an outwardly convex smooth hypersurface $\Sigma^{n} \subset X$ from which the exponential map is a diffeomorphism from the normal bundle to the outside, we find from the associated geodesic defining function $\tilde{r}=e^{-d_{\Sigma}}$, where $d_{\Sigma}$ is the signed geodesic distance from $\Sigma$, that the horospherical metric on $\Sigma=\{\tilde{r}=1\}$ is given by $h \in[\hat{g}]$. Hence, in the context of conformal geometry one may regard horospherical metrics associated to the hypersurfaces given in [5] simply as conformal representatives of the conformal infinity.

Now we would like to illustrate that the ambient metric construction in [8] somehow gives a nice extension to the notions of the horospherical metrics in [5]. As in [8], given a hyperbolic Poincaré manifold $\left(X^{n+1}, g\right)$ with conformal infinity $(M,[\hat{g}])$, we define the metric bundle $\mathcal{G}$ over $M$ to be the space of pairs $(h, x)$ with $x \in M$ and $h=s^{2} \hat{g}(x)$ for some $s \in \mathbb{R}_{+}$. The metric bundle $\mathcal{G}$ assumes the role of the light cone, that is, the space of horospheres, and the metric bundle comes with a tautological degenerate metric, $g_{\mathcal{G}}=\pi^{*} h$ at $z=(h, x)$, where $\pi$ is the bundle projection.

Fixing a representative $g_{0}$ of the conformal infinity $(M,[\hat{g}])$, one obtains a trivialization of metric bundle $\mathcal{G} \cong \mathbb{R}_{+} \times M$ by identifying

$$
(t, x) \in \mathbb{R}_{+} \times M \quad \text { with } \quad\left(t^{2} g_{0}(x), x\right) \in \mathcal{G} .
$$

Given local coordinates $(x)=\left(x^{1}, \ldots, x^{n}\right)$ on $\mathcal{U} \subset M$ we obtain local coordinates $(t, x)$ on $\pi^{-1}(\mathcal{U})$ where

$$
g_{i j}^{\mathcal{G}}=t^{2} g_{i j}^{0} d x^{i} d x^{j}
$$


so that the representative $g_{0}$ of the conformal infinity of $(X, g)$ can be considered as the section of the bundle $\mathcal{G} \cong \mathbb{R}_{+} \times M$ determined by the level submanifold $\{t=1\}$. On the ambient space $\left(\mathbb{R}_{+} \times M\right) \times \mathbb{R}$ with coordinates $(t, x, \rho)$, the ambient or cone metric $\tilde{g}=s^{2} g-d s^{2}$ with $X=\{s=1\}$ takes the normal form

$$
\tilde{g}=2 \rho d t^{2}+2 t d t d \rho+t^{2} g_{\rho},
$$

where

$$
-2 \rho=r^{2}, \quad s=r t \quad \text { for } \rho \leq 0,
$$

and $r$ is the geodesic defining function uniquely associated to $g_{0}$. Therefore, given an outwardly convex hypersurface $\Sigma^{n} \subset X^{n+1}$ and letting $\alpha=d_{\Sigma}$ denote the signed geodesic distance from $\Sigma$, which is positive outside $\Sigma$, one finds that under the change of variables

$$
t=e^{\alpha}
$$

the ambient metric restricted to $X$ takes the form

$$
\left.\tilde{g}\right|_{X}=d \alpha^{2}+e^{2 \alpha} g_{\alpha}
$$

Hence, one may view the change of variables $t=e^{\alpha}$ with respect to a given hypersurface as straightening out the hypersurface and giving a new coordinate on the metric bundle, which results in determining a new representative of the conformal infinity.

\section{REFERENCES}

[1] M. Anderson. $L^{2}$ Curvature and Volume Renormalization of AHE Metrics on 4-Manifolds. Math. Res. Lett. 8: 171-188, 2001. MR.1825268 (2002k:53079)

[2] Vincent Bonini, Pengzi Miao and Jie Qing. Ricci Curvature Rigidity for Weakly Asymptotically Hyperbolic Manifolds. Comm. Anal. Geom. 14(3): 603-612, 2006. MR 2260724 (2007f:53041)

[3] Sun-Yung Alice Chang, Jie Qing and Paul Yang. On the Topology of Conformally Compact Einstein 4-Manifolds. Noncompact problems at the intersection of geometry, analysis, and topology, 49-61, Contemp. Math., 350, Amer. Math. Soc., Providence, RI, 2004. MR2082390 (2005g:53075)

[4] Charles L. Epstein. Envelopes of Horospheres and Weingarten Surfaces in Hyperbolic 3-Space, unpublished preprint.

[5] José M. Espinar, José A. Gálvez and Pablo Mira. Hypersurfaces in $\mathbb{H}^{n+1}$ and Conformally Invariant Equations: The Generalized Christoffel and Nirenberg Problems. Jour. European Math. Soc. 11: 903-939, 2009. MR2538508

[6] C. Robin Graham. Volume and Area Renormalizations for Conformally Compact Einstein Metrics. The Proceedings of the 19th Winter School "Geometry and Physics" (Srni, 1999). Rend. Circ. Mat. Palermo (2) Suppl. No. 63: 31-42, 2000. MR1758076 (2002c:53073)

[7] Charles Fefferman and C. Robin Graham. Conformal Invariants. Asterisque 95-116, 1985. MR837196 (87g:53060)

[8] Charles Fefferman and C. Robin Graham. The Ambient Metric. math.DG: arXiv:0710.0919

[9] C. Robin Graham and John M. Lee. Einstein Metrics with Prescribed Conformal Infinity on the Ball. Adv. Math. 87(2): 186-225, 1991. MR1112625 (92i:53041)

[10] P. Guan and G. Wang. A Fully Nonlinear Conformal Flow on Locally Conformally Flat Manifolds. J. Reine Angew. Math. 557: 219-238, 2003. MR1978409 (2004e:53101)

[11] M. Gursky and J. Viaclovsky. Prescribing Symmetric Functions of the Eigenvalues of the Ricci Tensor. Annals of Mathematics (2) 166(2): 475-531, 2007. MR22373147 (2008k:53068)

[12] John M. Lee. The Spectrum of an Asymptotically Hyperbolic Einstein Manifold. Comm. Anal. Geom. 3(1-2): 253-271, 1995. MR.1362652(96h:58176)

[13] A. Li and Y.Y. Li. On Some Conformally Invariant Fully Nonlinear Equations. Comm. Pure Appl. Math. 56: 1416-1464, 2003. MR.1988895 (2004e:35072) 
[14] Jie Qing. On the Rigidity for Conformally Compact Einstein Manifolds. Int. Math. Res. Not. 21: 1141-1153, 2003. MR1962123 (2004a:53049)

[15] J. M. Schlenker. Hypersurfaces in $\mathbb{H}^{n}$ and the Space of Its Horospheres. Geom. Anal. Funct. Anal. 12: 395-435, 2002. MR1911666 (2003d:53108)

Department of Mathematics, California Polytechnic State University, San Luis Obispo, CALifornia 93407

E-mail address: vbonini@calpoly.edu

Departmento de Geometría y Topología, Universidad de Granada, E-18071 Granda, SPAIN

E-mail address: jespinar@ugr.es

Department of Mathematics, University of California, Santa Cruz, California 95064

E-mail address: qing@ucsc.edu 